\title{
Aircraft Conflict Prediction and Resolution using Brownian Motion ${ }^{1}$
}

\author{
Jianghai $\mathrm{Hu}$, John Lygeros, Maria Prandini and Shankar Sastry \\ Department of Electrical Engineering and Computer Sciences \\ University of California, Berkeley CA 94720 \\ $\{j$ ianghai, lygeros, prandini, sastry\}@robotics.eecs.berkeley.edu
}

\begin{abstract}
The probability of conflict between two aircraft is calculated by modeling aircraft motion as a deterministic trajectory plus a (scaled) Brownian motion perturbation. In this formalism, the probability of conflict becomes the probability that a Brownian motion escapes from a time-varying safe region. Approximate expressions for the probability of conflict are obtained in closed form for both the finite and infinite horizon cases. Based on these expressions, an autonomous algorithm is proposed for decentralized conflict resolution. The algorithm generalizes potential field ideas to path planning in a highly dynamic environment.
\end{abstract}

\section{Introduction}

Despite technological advances such as relatively inexpensive on-board computers and global positioning systems, the current Air Traffic Management System (ATMS) remains a bottleneck under the dramatically increasing demand for air travel in recent years. It is believed that by improving the efficiency of current semiautomated ATMS, the increased demand in air traffic can be handled in a more reliable way, without resorting to such costly options as building larger airports or adding more runways. The notion of free flight has been proposed to shift the totally centralized decision making in the current ATMS to individual aircraft, in a hope to alleviate the burden of central controller and decrease travel times, unexpected delays, and fuel consumption. [1] highlights the hierarchical and hybrid control issues associated with free flight.

Perhaps the most important goal of the new ATMS is to improve the safety, which is typically characterized in terms of the number of conflicts (collisions and near misses). For the current system, a conflict is defined as a situation where two aircraft come within 5 nauti-

\footnotetext{
${ }^{1}$ Research supported by DARPA under grant F33615-98-C3614 , by NASA under grant NAG 2-1039, by ARO under grant MURI DAAH 04-96-1-0341 and by the California PATH project under MOU 312 .
}

$0-7803-5250-5 / 99 / \$ 10.00$ 두 1999 IEEE cal miles (nmi) of one another horizontally and within 1000 or 2000 feet $(\mathrm{ft})$ vertically, depending on whether the flight level is below or above $29,000 \mathrm{ft}$ respectively (see [2]). Conflict avoidance typically consists of two stages: conflict detection and conflict resolution. In the conflict detection stage, the motions of aircraft are predicted based on their positions, headings and flight plans, and conflict situations are identified. This information is then used in the resolution stage to re-plan the trajectories for the aircraft involved in the conflicts.

The major contributor to the uncertainty in the aircraft motion is wind, for which a consistent physical model is not available yet. However, since the prediction error can be modeled as the sum of a large number of independent random perturbations in disjoint time intervals, it is expected to be Gaussian. This hypothesis was indeed verified by empirical data in [2], which also suggested that the uncertainty can be decomposed into two components: an along track component whose variance grows with time, and a cross track component whose variance remains roughly constant. Based on this model, a number of methods have been proposed to predict the probability of conflict (PC) for aircraft pairs over horizons of the order of 20 minutes. In [2] the notion of uncertainty ellipse is used to obtain a closed form over-approximation for PC. In [3], a randomized estimation algorithm is proposed for computing $\mathrm{PC}$. Finally, [4] uses Monte Carlo simulation to determine PC for typical encounters.

In this paper we give an alternative treatment by modeling the perturbation as a (scaled) Brownian motion (BM). We focus on the two dimensional (2-D) case, assuming that both aircraft fly at the same altitude. Intuitively, the probability of conflict is the proportion of sample paths leading to a collision among all possible paths. BM gives us a measure of the probability of each path, where paths of large and steady deviation are less likely than paths of small and fluctuating deviation. One major advantage of our approach is that it provides closed form formulae (though approximations), which not only makes its implementation computationally inexpensive, but also enables us to easily 


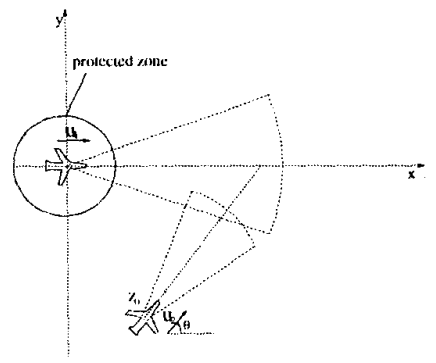

Figure 1: Typical encounter situation.

derive a resolution algorithm. A similar model has been employed in [5] for highway safety analysis.

We start by presenting the basic model used in our computation in Section 2. In Section 3 we derive closed form approximations of $\mathrm{PC}$ for typical encounters. These results are then used in Section 4 to derive a decentralized conflict resolution algorithm.

\section{Model}

Consider two aircraft, labeled 1 and 2, moving on the same horizontal plane. Assume without loss of generality that at time $t=0$, aircraft 1 is at the origin of a global coordinate frame, flying from left to right with a velocity $\vec{u}_{1}=(u, 0)^{T} \in \Re^{2}$, while aircraft 2 is at position $\vec{z}_{0} \in \Re^{2}$, flying with a velocity $\vec{u}_{2} \in \Re^{2}$ which makes an angle $\theta$ with $\vec{u}_{1}$ (Figure 1 ). We refer to aircraft 2 as the intruder. A conflict occurs if the intruder enters the protected zone of radius $5 \mathrm{nmi}$ around aircraft 1 or vice versa.

For the positions $\vec{z}_{1}(t)$ and $\vec{z}_{2}(t)$ of the two aircraft, we propose a kinematic model of the following form:

$$
\begin{aligned}
& \vec{z}_{1}(t)=\vec{u}_{1} t+\Sigma \vec{B}_{1}(t) \\
& \vec{z}_{2}(t)=\vec{z}_{0}+\vec{u}_{2} t+T(\theta) \Sigma \vec{B}_{2}(t)
\end{aligned}
$$

where $\Sigma=\operatorname{diag}\left(\sigma_{a}, \sigma_{c}\right) . \quad \sigma_{a}, \sigma_{c}$ model the variance growth rate in the along track and cross track component respectively $\left(\sigma_{a}>\sigma_{c}\right.$ typically). $T(\theta)$ is the matrix corresponding to a counterclockwise rotation by $\theta$. $\vec{B}_{1}(t)$ and $\vec{B}_{2}(t)$ are independent standard 2-D BM's.

Subtracting (1) from (2) leads to

$$
\Delta \vec{z}(t)=\vec{z}_{0}+\Delta \vec{u} \cdot t-\vec{W}(t),
$$

where $\Delta \vec{z}(t) \triangleq \vec{z}_{2}(t)-\vec{z}_{1}(t), \Delta \vec{u} \triangleq \vec{u}_{2}-\vec{u}_{1}$, and $\vec{W}(t) \triangleq$ $\Sigma \vec{B}_{1}(t)-T(\theta) \Sigma \vec{B}_{2}(t)$. Equation (3) suggests that one can think of the motion of aircraft 1 as consisting only of the perturbation $\vec{W}(t)$, and the motion of aircraft 2 as deterministic with constant velocity $\Delta \vec{u}$ starting

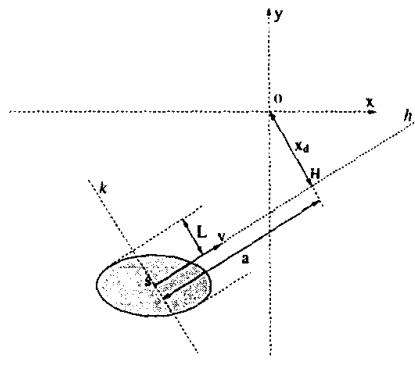

Figure 2: Transformed protection zone.

from $\vec{z}_{0}$. Furthermore, there exists a nonsingular matrix $P$ such that $P^{-1} \vec{W}(t)$ is a standard 2-D BM. By the definition of $\vec{W}(t)$, we have that for all $s, t \geq 0$,

$$
E\left[\vec{W}(t+s) \vec{W}^{T}(t)\right]=\left(\Sigma^{2}+T(\theta) \Sigma^{2} T(\theta)^{T}\right) t .
$$

Note that $\Sigma^{2}+T(\theta) \Sigma^{2} T(\theta)^{T}$ can be decomposed as $P P^{T}$ where $P=\sqrt{2} T\left(\frac{\theta}{2}\right) \Lambda$ and $\Lambda=\operatorname{diag}\left(\lambda_{1}, \lambda_{2}\right)$ with:

$$
\left\{\begin{array}{l}
\lambda_{1}=\sqrt{\sigma_{a}^{2} \cos ^{2}\left(\frac{\theta}{2}\right)+\sigma_{c}^{2} \sin ^{2}\left(\frac{\theta}{2}\right)} \\
\lambda_{2}=\sqrt{\sigma_{a}^{2} \sin ^{2}\left(\frac{\theta}{2}\right)+\sigma_{c}^{2} \cos ^{2}\left(\frac{\theta}{2}\right)}
\end{array}\right.
$$

If we define $\vec{n}(t) \triangleq P^{-1} \vec{W}(t)$, we in fact have $E[\vec{n}(t+$ s) $\left.\vec{n}^{T}(t)\right]=t I$, i.e., $\vec{n}(t)$ is a standard 2-D BM.

Therefore, by performing the transformation $P^{-1}$, we can assume that the motion of aircraft 1 is a standard 2-D BM starting from the origin, while the motion of aircraft 2 is of constant velocity $\vec{v}=\left(v_{1}, v_{2}\right)^{T}$ starting from $\vec{s}=\left(s_{1}, s_{2}\right)^{T}$ (see Figure 2), where

$$
\vec{s}=P^{-1} \vec{z}_{0}, \quad \vec{v}=P^{-1} \Delta \vec{u} .
$$

Define $x_{d}$ as the distance from the origin to the line $h$ along which aircraft 2 is flying, and $a$ as the distance from aircraft 2 at $t=0$ to the projection $H$ of the origin on $h$. Then, in the new coordinate system:

$$
x_{d}=\frac{\left|s_{1} v_{2}-s_{2} v_{1}\right|}{\sqrt{v_{1}^{2}+v_{2}^{2}}}, \quad a=-\frac{s_{1} v_{1}+s_{2} v_{2}}{\sqrt{v_{1}^{2}+v_{2}^{2}}} .
$$

Ignore the noise temporarily. Then, in the new coordinate system, a positive $a$ indicates that the two aircraft are approaching each other and the minimal separation during the encounter is $x_{d}$. On the other hand, a negative $a$ indicates that they are flying away from each other and the minimal separation occurs at time $t=0$. Moreover, the circular protection zone of radius $R=5$ $\mathrm{nmi}$ around aircraft 2 is transformed into an ellipse centered initially at $\vec{s}$ and with boundary described by:

$$
\lambda_{1}^{2}\left(x-s_{1}\right)^{2}+\lambda_{2}^{2}\left(y-s_{2}\right)^{2}=R^{2} / 2,
$$

moving along with aircraft 2. A conflict occurs if and only if the 2-D BM (i.e., aircraft 1 in the new coordinate system) ever wanders into this moving ellipse. 
The model proposed here assumes that the initial positions of the aircraft are known precisely, i.e., it ignores errors in the radar and GPS measurements. This can be justified by the fact that uncertainty in the initial position will become rapidly dominated by the perturbation to the aircraft motion as time goes on (see [4]). Another assumption we make is that the perturbations to the motions of different aircraft are independent. This assumption, though inaccurate, is commonly made in the literature (see $[2,3])$.

Remark: In the model of [2], the cross track variance saturates once it reaches a fixed value (typically 0.5-1 $\mathrm{nmi}^{2}$ ) and the along track variance grows quadratically in time. By comparison, in our model both of them grow linearly with time. On the other hand, we intend to use our model for short term conflict detection and resolution in a free flight scenario. Therefore, since the saturation is mainly due to pilot feedback, it may not have enough time to occur over short time horizons. Moreover, for free flight each aircraft has only partial information about the intentions of their neighbors. In this situation, there is no reason to assume that the intruder will make an effort to maintain its current heading precisely. Finally, the quadratically increasing along track variance in [2] is largely due to "global" wind effects which, although unknown, remain constant and affect the motion of both aircraft in a similar way over the relatively short conflict horizon. Therefore, they tend to cancel out in the relative position. The perturbations in (1) and (2) model mainly local wind effects such as air turbulence as well as deviations due to mechanical and human factors for each aircraft.

\section{Conflict Prediction}

Following [1], we distinguish three different kinds of conflict of increasing generality, and derive the probability of conflict (PC) for each particular case.

1. Overtake: Two aircraft fly along the same path with the faster one trailing the slower one.

2. Collision: Two aircraft fly along crossing paths and exact collision is predicted.

3. Near miss: Similar to collision, except that "exact collision" is replaced by "approach within $5 \mathrm{nmi".}$

\subsection{PC for Overtake}

Suppose that aircraft 1 and 2 are both flying from left to right along the $\mathrm{x}$ axis, with an initial distance $\Delta x$ and aircraft 1 following aircraft 2 at a higher speed $u_{1}>u_{2}$. In this setting, equation (3) simplifies to:

$$
\left\{\begin{array}{l}
\Delta x(t)=\Delta x+\Delta u t+\sigma_{1} B_{x}(t) \\
\Delta y(t)=\sigma_{2} B_{y}(t)
\end{array}\right.
$$

where $(\Delta x(t), \Delta y(t))$ is the relative position of aircraft 2 with respect to aircraft 1 at time $t, \Delta u \triangleq u_{2}-u_{1}, B_{x}(t)$, $B_{y}(t)$ are independent standard BM's, and $\sigma_{1}=\sqrt{2} \sigma_{a}$, $\sigma_{2}=\sqrt{2} \sigma_{\mathrm{c}}$ are the combined variances of the perturbations of both aircraft (assuming independence).

A coordinate transformation allows us to adopt the viewpoint that the motion of aircraft 1 is a standard 2-D BM $B_{t}$ starting from the origin, while aircraft 2 is moving at constant speed $v=|\Delta u| / \sigma_{1}$ to the left, with an initial distance $a=\Delta x / \sigma_{1}$ from aircraft 1 . In the new coordinate system, the protected zone is transformed into an ellipse with axes $L=R / \sigma_{2}$ and $S=R / \sigma_{1}$ moving along with aircraft 2 (Figure 3 ).

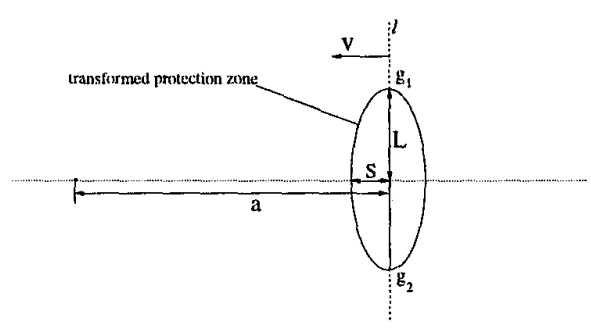

Figure 3: Transformed protection zone.

A conflict occurs if and only if $B_{t}$ ever wanders into the moving ellipse. Denote this event as $F$. The probability $P(F)$ does not admit a closed form formula. However we can approximate it by a "decoupled" event. Denote by $\tau$ the first time $B_{t}$ hits the vertical line $l$ and define $A \triangleq\left\{\left|B_{y}(\tau)\right| \leq L\right\}$. Since $A \subseteq F, P(A) \leq P(F)$. However since usually $\Delta u$ is much larger than the rate of the BM, and because of the shape of the ellipse, it can be shown (see [6]) that $P(F) \simeq P(A)$.

By the Bachelier-Levy theorem (see [7]), $\tau$ has density:

$$
p_{\tau}(t)=\frac{a}{\sqrt{2 \pi t^{3}}} \exp \left(-\frac{(a-v t)^{2}}{2 t}\right) .
$$

So the approximate probability of conflict is:

$$
\begin{aligned}
P(A) & =\int_{0}^{\infty} p_{\tau}(t) \int_{|y|<L} \frac{1}{\sqrt{2 \pi t}} \exp \left(-\frac{y^{2}}{2 t}\right) d t \\
& =1-2 \int_{0}^{\infty} p_{\tau}(t) Q\left(\frac{L}{\sqrt{t}}\right) d t
\end{aligned}
$$

where $Q(x) \triangleq \int_{x}^{\infty} \frac{1}{\sqrt{2 \pi}} \exp \left(-t^{2} / 2\right) d t$.

Notice that $E[\tau]=a / v$. In case $v$ is larger than 1 or equivalently $|\Delta u|>\sigma_{1}, p_{\tau}(t)$ concentrates near $t_{0} \triangleq$ $a / v=\Delta x /|\Delta u|$. Expand $q(t) \triangleq Q(L / \sqrt{t})$ about $t_{0}$ to get:

$$
q(t) \simeq q\left(t_{0}\right)+q^{\prime}\left(t_{0}\right)\left(t-t_{0}\right)+\frac{1}{2} q^{\prime \prime}\left(t_{0}\right)\left(t-t_{0}\right)^{2} .
$$

By substituting (8) into (7) (see [6] for the details), we derive the approximation: 

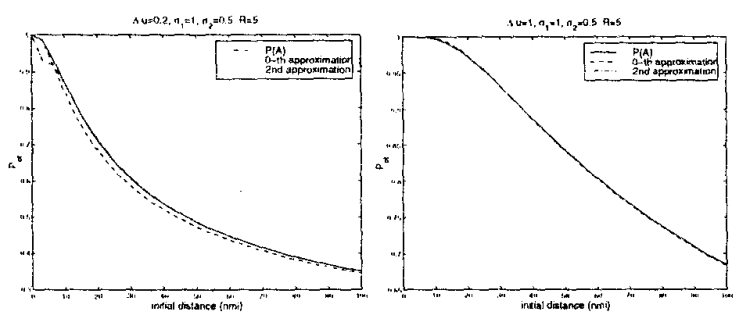

Figure 4: Plot of $P_{o t}$ (Left: $\Delta u=0.2 \mathrm{nmi} / \mathrm{min}$, Right: $\Delta u=1 \mathrm{nmi} / \mathrm{min}$ )

Assertion 1 PC for overtake can be approximated by:

$$
P_{o t} \triangleq 1-2 \cdot Q\left(\frac{L}{\sqrt{t_{0}}}\right)-\frac{K}{4 v^{2} \sqrt{2 \pi}} \exp \left(-\frac{L^{2}}{2 t_{0}}\right),
$$

where $t_{0}=\Delta x /|\Delta u|$ and $K=L^{3} / \sqrt{t_{0}^{5}}-3 L / \sqrt{t_{0}^{3}}$. If only the 0 -th order term is used in (8), then

$$
P_{o t} \simeq 1-2 \cdot Q\left(L / \sqrt{t_{0}}\right)
$$

In Figure $4, P(A)$ computed by numerical integration and its 0 -th and 3 -rd order approximation are plotted as functions of $\Delta x$, for the two cases $\Delta u=0.2 \mathrm{nmi} / \mathrm{min}$ and $\Delta u=1 \mathrm{nmi} / \mathrm{min}$. Here $\sigma_{1}=1, \sigma_{2}=0.5$ (whose squares are in $\mathrm{nmi}^{2} / \mathrm{min}$, which is omitted from now on for simplicity), and $R=5 \mathrm{nmi}$. From these plots, it can be seen that Assertion 1 gives us a remarkably sharp estimate of $P(A)$ (and hence $P(F)$ ) even in the case when $v$ is much smaller than 1 (the approximation error when $v>1$ is barely visible).

Sometimes it is more reasonable to limit the prediction to a finite horizon, since a situation where $\mathrm{PC}$ is nearly 1 but with a projected collision time $\bar{t}$ of 30 minutes may not be as dangerous as a situation with a smaller PC but $\bar{t}=15$ min. Instead of simply weighting $\mathrm{PC}$ and $\bar{t}$, we can alternatively compute $\mathrm{PC}$ for the overtake case within a fixed horizon $t_{f}$ as:

$$
\begin{aligned}
P_{o t}^{t_{f}}(A) & =\int_{0}^{t_{f}} p_{\tau}(t) \int_{|y|<L} \frac{1}{2 \pi t} \exp \left(-\frac{y^{2}}{2 t}\right) d t \\
& =\int_{0}^{t_{f}} p_{\tau}(t)\left[1-2 Q\left(\frac{L}{\sqrt{t}}\right)\right] d t .
\end{aligned}
$$

Following a procedure similar to that used for the infinite horizon case (see [6]), we get the approximation:

Assertion $2 P C$ within time $t_{f}$ for overtake can be approximated by:

$$
\begin{aligned}
P_{o t}^{t_{f}} & \triangleq Q\left(\frac{a-v t_{f}}{\sqrt{t_{f}}}\right) G_{1}+e^{2 a v} Q\left(\frac{a+v t_{f}}{\sqrt{t_{f}}}\right) G_{2} \\
& +2 q^{\prime \prime}\left(t_{0}\right) \frac{a \sqrt{t_{f}}}{v^{2} \sqrt{2 \pi}} \exp \left(-\frac{\left(a-v t_{f}\right)^{2}}{2 t_{f}}\right)
\end{aligned}
$$
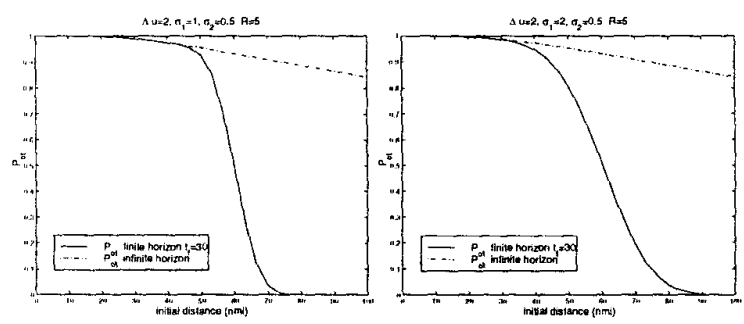

Figure 5: $P_{o t}^{t_{f}}$ with $t_{f}=30 \mathrm{~min}, \Delta u=2 \mathrm{nmi} / \mathrm{min}$. Left: $\sigma_{1}=1$, Right: $\sigma_{1}=2$.

where

$$
\begin{aligned}
& G_{1} \triangleq 1-2 q\left(t_{0}\right)-q^{\prime \prime}\left(t_{0}\right) a / v^{3}, \\
& G_{2} \triangleq 1-2 q\left(t_{0}\right)+4 q^{\prime}\left(t_{0}\right) t_{0}-q^{\prime \prime}\left(t_{0}\right)\left(4 t_{0}^{2}-a / v^{3}\right) .
\end{aligned}
$$

Figure 5 shows the comparison between $P_{\text {ot }}$ and $P_{\text {ot }}^{t_{f}}$, $t_{f}=30 \mathrm{~min}$, as functions of $\Delta x$ for two different values of $\sigma_{1}\left(\sigma_{1}=1\right.$ and $\left.\sigma_{1}=2\right)$, with $\sigma_{2}=0.5$ and $R=5$ nmi. Notice that $P_{o t}^{t_{f}}$ coincides with $P_{\text {ot }}$ for small $\Delta x$, but drops to zero at around $\Delta u t_{f}$. The larger the $\sigma_{1}$, the slower the drop.

\subsection{PC for Collision and Near Miss}

We now deal with the case when the paths of the two aircraft cross at an arbitrary angle $\theta$. In the following discussion we use the concepts introduced in Section 2. In particular, the circular protected zone of radius $R$ is transformed into an ellipse centered around aircraft 2 , with initial boundary determined by equation (5).

A conflict occurs if and only if the 2-D BM wanders into this moving ellipse. In the spirit of the previous subsection, we calculate the projected width $L$ of the ellipse along the direction of $\vec{v}$ as (see Figure 2):

$$
L=\frac{R}{\lambda_{1} \lambda_{2}} \sqrt{\frac{v_{1}^{2} \lambda_{1}^{2}+v_{2}^{2} \lambda_{2}^{2}}{2\left(v_{1}^{2}+v_{2}^{2}\right)}} .
$$

In this case, we estimate $\mathrm{PC}$ by approximating the event of conflict as the event that the first time the 2-D BM hits line $k$, it is within a distance of $L$ from the center of the ellipse. Although the ellipse may be far from symmetrical with respect to the direction of $\vec{v}$, the error introduced in our approximation is partly reduced due to the shape of the ellipse on different sides of the trajectory. For a more formal validation, see [6].

By some rotation, we can assume that $\vec{v}$ is aligned with the positive $x$ axis. The time $r$ for aircraft 1 to reach the line $k$ has density of the form (6) with $v=|\vec{v}|$ being the norm of $\vec{v}$ defined in (4). Assume $a>0$. As for the overtake case, we have the following approximation of 

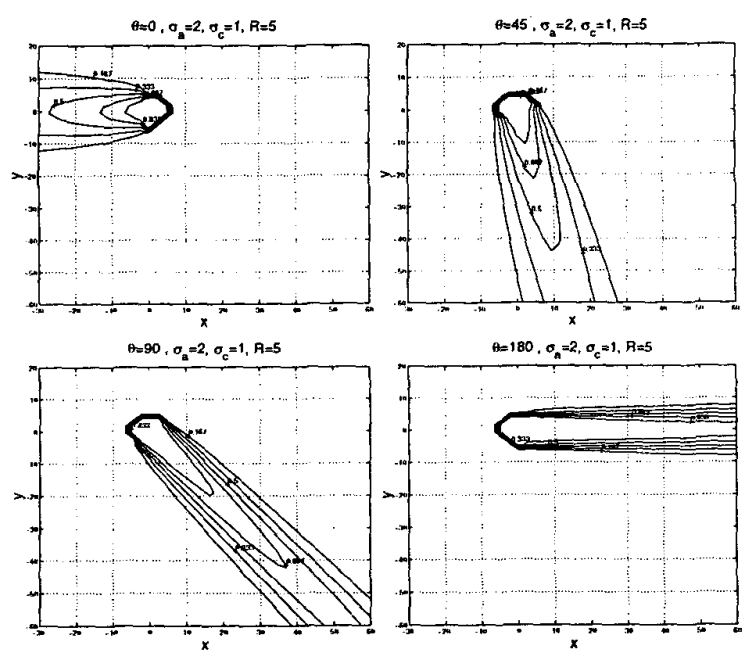

Figure 6: General collision $P_{n m}$ for different path angles.

PC for collision and near miss:

$$
\begin{aligned}
P_{n m} & =\int_{0}^{\infty} p_{\tau}(t) \int_{\left|y-x_{d}\right|<L} \frac{1}{2 \pi t} \exp \left(-\frac{y^{2}}{2 t}\right) d t \\
& =\int_{0}^{\infty} p_{\tau}(t)\left[Q\left(\frac{x_{d}-L}{\sqrt{t}}\right)-Q\left(\frac{x_{d}+L}{\sqrt{t}}\right)\right] d t .
\end{aligned}
$$

A procedure similar to the one used in the overtake case provides closed form approximations for (9). For example, if the 0 -th order term is used, we get:

Assertion $3 P C$ for collision and near miss can be approximated by $\left(t_{0}=a / v\right)$ :

$$
P_{n m} \simeq Q\left(\frac{x_{d}-L}{\sqrt{t_{0}}}\right)-Q\left(\frac{x_{d}+L}{\sqrt{t_{0}}}\right) .
$$

Higher order approximation and the finite horizon version $P_{n m}^{t_{f}}$ can also be obtained similarly (see [6]).

Figure 6 shows some level curves of $P_{n m}$ as a function of the initial position $\vec{z}_{0}$ of aircraft 2 for the path angles $\theta=0^{\circ}, 45^{\circ}, 90^{\circ}, 180^{\circ}$. In each subplot, aircraft 1 is moving from left to right at velocity $u_{1}=7 \mathrm{nmi} / \mathrm{min}$ starting from the origin and aircraft 2 is moving at velocity $u_{2}=8 \mathrm{nmi} / \mathrm{min}$, but $\theta$ assumes a different value. To see the results more compactly, we used relatively large $\sigma_{a}$ and $\sigma_{c}\left(\sigma_{a}=2, \sigma_{c}=1\right)$ and $R=5 \mathrm{nmi}$. It can be seen that as the path angle $\theta$ gets larger and larger, the region delimited by the same $P_{n m}$ equi-probability line gets more extended. For the case $\theta=90^{\circ}$, Figure 7 shows the level curves of $P_{n m}^{t_{f}}$ with $t_{f}=10,15$ min, which look like a truncation of the corresponding infinite horizon version. If $\sigma_{a}$ and $\sigma_{c}$ increase, the truncation becomes smoother.
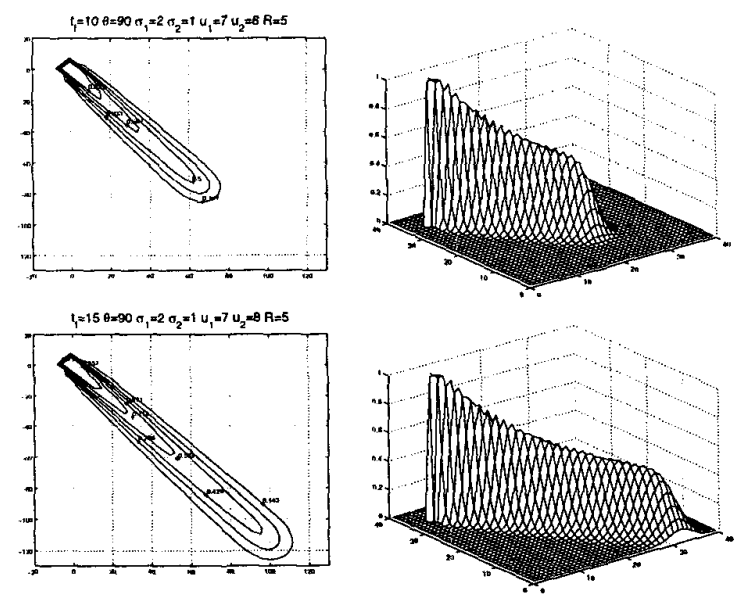

Figure 7: General collision $P_{n m}^{t_{f}}$ for different $t_{f}$.

\section{Conflict Resolution}

In this section, we introduce a decentralized conflict resolution algorithm which exploits PC calculated in (10) to guide each aircraft to its desired destination, while avoiding conflicts with other aircraft flying nearby. Although we deal specifically with ATMS, the following discussion regards the general issue of path planning for multiple agents moving in a dynamic environment.

In [8], an algorithm for aircraft conflict resolution is proposed based on the potential and vortex field method. However, since the potential field defined in [8] depends only on the distance, the generated avoidance maneuvers sometimes contain abrupt - and hence non-flyable - turns. Since PC derived in (10) contains information about both distance and relative velocity, it is expected to be a better candidate for formulating a conflict resolution algorithm.

Consider first the case when two aircraf start from positions $\vec{z}_{1}(0)$ and $\vec{z}_{2}(0)$ and have destinations $\vec{d}_{1}$ and $\vec{d}_{2}$ respectively. Assume their initial heading is toward their own destination and they fly at a constant speed, say $u_{1}$ and $u_{2}$ respectively. At each time instant t, $P_{n m}(t)$ can be calculated based on the aircraft positions $\vec{z}_{1}(t), \vec{z}_{2}(t)$ and velocities $\vec{u}_{1}(t), \vec{u}_{2}(t)$.

We define for each aircraft three headings of interest:

- Current heading $\theta_{c}$ : Direction along which the aircraft is currently flying.

- Destination heading $\theta_{d}$ : Direction defined by the current aircraft position and its destination.

- Gradient heading $\theta_{g}$ : Direction corresponding to the highest decrease of $\mathrm{PC}$, i.e., the direction determined by the negative gradient of $P_{n m}$ as a 

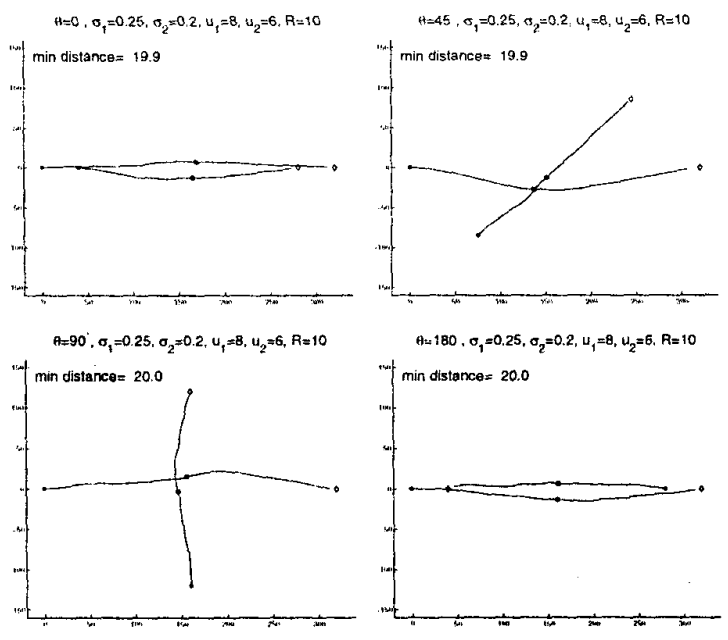

Figure 8: Resolution for two aircraft encounters.

function only of the current aircraft position.

The proposed resolution scheme is quite simple: at each time step, each aircraft updates its own heading as:

$$
\theta_{\text {next }}= \begin{cases}\bar{\theta} & \text { if }\left|\vec{\theta}-\theta_{c}\right|<\beta \\ \theta_{c}+\beta \operatorname{sgn}\left(\bar{\theta}-\theta_{c}\right) & \text { otherwise, }\end{cases}
$$

where $\beta$ is the maximal allowed turn angle per time step, and $\bar{\theta}=P_{n m} \theta_{g}+\left(1-P_{n m}\right) \theta_{d}$. Intuitively, if $P_{n m}$ is high, then decreasing $P_{n m}$ becomes a priority, hence $\theta_{g}$ should be pursued more. If instead, $P_{n m}$ is negligible, then $\theta_{c}$ should be pursued. In any case, due to the nonholonomic nature in aircraft motion, the deviation of the new heading from $\theta_{c}$ should not exceed $\beta$.

Simulation results are shown in Figure 8 for different cross path angles with $u_{1}=8 \mathrm{nmi} / \mathrm{min}$ and $u_{2}=6$ $\mathrm{nmi} / \mathrm{min}$. To make the resolution maneuvers more evident, we chose a relatively large $R(R=10 \mathrm{nmi})$. The time step is set equal to $0.5 \mathrm{~min}$. $\sigma_{a}=0.25$ and $\sigma_{c}=0.2$ are used both in the calculation of $P_{n m}$ and in the generation of noise for the simulated trajectories. In each subplot of Figure 8, the starting point is marked with a star, the destination with a diamond, whereas a circle marks the point where the minimal separation occurs.

Following a similar procedure, it is possible to extend our algorithm to the encounter of more than two aircraft. Simulation results for different three aircraft encounter situations are shown in Figure 9. In each case, we assume the most severe situation, i.e., there is a predicted exact collision sometime in the future involving all three aircraft whose speeds are $u_{1}=8 \mathrm{nmi} / \mathrm{min}$, $u_{2}=10 \mathrm{nmi} / \mathrm{min}$, and $u_{3}=6 \mathrm{nmi} / \mathrm{min}$, respectively.

Notice that in all the cases, simulations indicate that the safety separation requirement is satisfied. We are
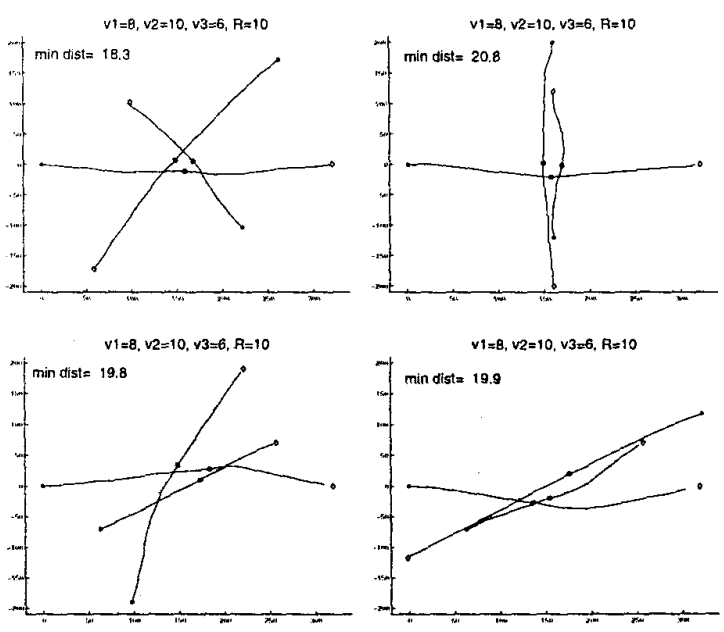

Figure 9: Resolution for three aircraft encounters.

currently working on a formal proof of the safety of the proposed resolution algorithm in a suitable probabilistic setting.

\section{References}

[1] S. Sastry, G. Meyer, C. Tomlin, J. Lygeros, D. Godbole, and G. Pappas, "Hybrid systems in air traffic control," in IEEE Conference on Decision and Control, pp. 1478-1483, 1995.

[2] R. Paielli and H. Erzberger, "Conflict probability estimation for free flight," Journal of Guidance, Control and Dynamics, vol. 20, no. 3, pp. 588-596, 1997.

[3] M. Prandini, J. Lygeros, A. Nilim, and S. Sastry, "A probabilistic framework for aircraft conflict detection," in AIAA Guidance, Navigation and Control, 1999. Accepted.

[4] L. Yang and J. Kuchar, "Prototype conflict alerting logic for free flight," in Proc. 35th AIAA Airspace Science Meeting $\&$ Exhibit, AIAA 97-0220, January 1997.

[5] J. Hu, J. Lygeros, M. Prandini, and S. Sastry, "A probabilistic framework for highway safety analysis," in IEEE Conference on Decision and Control, 1999.

[6] J. Hu, J. Lygeros, M. Prandini, and S. Sastry, "Case study of collision probability," PATH technical report, Institute of Transportation Study, UC Berkeley, to appear, 1999.

[7] H. R. Lerche, Boundary crossing of Brownian motion. Springer-Verlag, 1986.

[8] J. Kosecka, C. Tomlin, G. Pappas, and S. Sastry, "Generation of conflict resolution maneuvers for air traffic management," in Proc. IEEE Conf. on Intelligent Robotics and System '97, vol. 3, pp. 1598-1603, 1997. 